\title{
Atomic and Electronic Structure of Graphene-Oxide
}

\author{
K. A. Mkhoyan***, A. W. Contryman**, J. Silcox**, D. A. Stewart**, G. Eda***, C. Mattevi***, S. \\ Miller*** and, M. Chhowalla*** \\ *Department of Chemical Engineering and Materials Science and Engineering, University of Minnesota, \\ Minneapolis, MN 55455 \\ **School of Applied and Engineering Physics, Cornell University, Ithaca, New York 14853 \\ ***Department of Material Science and Engineering, Rutgers University, Piscataway, New Jersey 08854
}

Thin sheets of graphene oxide (GO) have recently emerged as a new carbon-based nanoscale material that also provides an alternative path to graphene [1]. The solubility of graphene oxide in water and other solvents allows it to be uniformly deposited onto wide ranging substrates in the form of thin films or networks, which makes it potentially useful for macroelectronics [2]. Graphene oxide is an insulator but controlled oxidation provides tunability of the electronic and mechanical properties including the possibility of accessing zero-band gap graphene via complete removal of the $\mathrm{C}-\mathrm{O}$ bonds. The structure of $\mathrm{GO}$ is often simplistically assumed to be a graphene sheet bonded to oxygen in the form of carboxyl, hydroxyl or epoxy groups. Here, we elucidate the atomic and electronic structure of GO using composition sensitive annular dark field (ADF) imaging of single and multilayer sheets and electron energy loss spectroscopy (EELS) for measuring the fine structure of the $\mathrm{C}$ and $\mathrm{O}$ K-edges as well as low-loss electronic excitations in a scanning transmission electron microscope (STEM).

ADF image of the several layers of the GO suspended over a hole is shown in Fig. 1(c). While the mono, bi-, and trilayered films can be imaged, the signal from the single layer was found to be very weak as indicated by the fact that it is barely distinguishable from the hole in the middle of the image. However, a high-magnification ADF image taken from this single sheet reveals not only the sheet but also the variation of intensities within the image. Fig. 1(d) is one such high-resolution image from a single layer GO. Strong variation of the ADF intensity in the single film suggests that the oxidation of the graphene is uniformly random throughout the sheet. Analysis of the ADF intensities from the dotted regions of Figure 1(c) reveals that the thicknesses of the mono-, bi-, and trilayers scale as 1:1.5:2.0, similar to the AFM results [3].

To study the electronic structure of GO films, core-level EELS spectra of C and O K-edges were measured from several single GO sheets. Fig. 2(a) shows the C K-edge recorded from GO film in and for comparison plotted with $\mathrm{C}$ K-edge spectra from amorphous-carbon $(\mathrm{a}-\mathrm{C})$ and graphite. A spectrum of the $\mathrm{O}$ $\mathrm{K}$-edge measured from the same sheet is presented in Fig. 2(b) and compared with corresponding spectrum in a- $\mathrm{SiO}_{2}$. The fine structure of the $\mathrm{C} \mathrm{K}$-edge in $\mathrm{GO}$ shows considerable differences relative to those in graphite and a-C. However, it also indicates the presence of $\sigma^{*}$ peak with energy close to that in graphite. To enhance the fine structure in $\mathrm{C}$ K-edge, the STEM probe function was removed from the data and the resulting spectrum is shown in Fig. 2(c). The fine structure of O K-edge, which lacks distinct features, indicates the absence of periodicity for the oxygen atoms in the film. Since there is no existing carbon-oxide in solid form, the $\mathrm{O}$ K-edge spectrum from GO sheets was compared with $\mathrm{O} \mathrm{K}$-edge of a-SiO${ }_{2}$, which also has an initial peak at $538 \mathrm{eV}$ and an extended broad second peak at about $560 \mathrm{eV}$. Strong similarities between the two are noticeable. These results are in agreement with ADF imaging showing random attachment of oxygen atoms on graphene sites during oxidation.

Electron energy loss spectroscopy combined with STEM-ADF imaging and AFM-depth profiling shows that graphene oxide films have substantially different density-of-states than those in graphene and a-C. It also indicates that $\mathrm{O}$ atoms attach to graphene sites randomly and convert $\mathrm{sp}^{2} \mathrm{C}$ bonds in graphene to $\mathrm{sp}^{3}$ bonds. While the structural modifications of graphene are dependent on the oxidation level, the results show 
that a ratio of 1:5 $\mathrm{O}$ to $\mathrm{C}$ atoms is sufficient to transform the measured $40 \%$ of the $\mathrm{C}$ bonds into $\mathrm{sp}^{3}$ bonds. As a consequence, the atomic structure of oxidized graphene is highly distorted, becoming a semiamorphous solid carbon oxide with undulations resulting in a surface roughness of about $0.6 \mathrm{~nm}$. These results are also supported by our ab initio calculations [4].

\section{References}

[1] S. Stankovich, et al., Nature 442, 282 (2006).

[2] G. Eda, G. Fanchini, and M. Chhowalla, Nat. Nanotechnol. 3, 270 (2008).

[3] K.A. Mkhoyan et al., Nano Lett. 9, 1058 (2009).

[4] This work is supported primarily by the NSF EEC-0117770 and NYSTAR C020071 and DMR 9632275 through Cornell University and NSF CAREER Award ECS 0543867. Authors would also like to acknowledge technical support of M. Thomas, L.F. Kourkoutis, J. Grazul, and G. Fanchini.

(a)

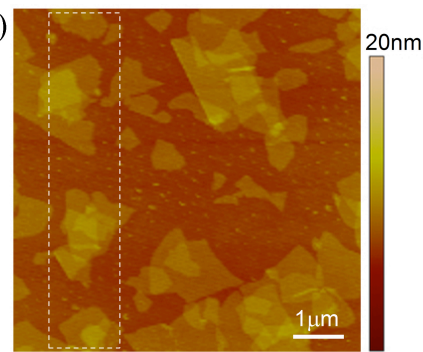

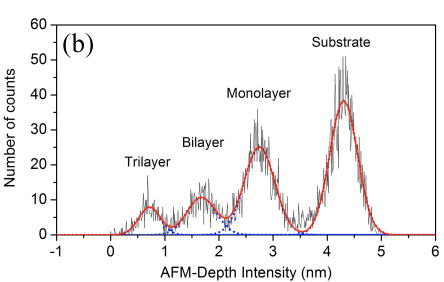
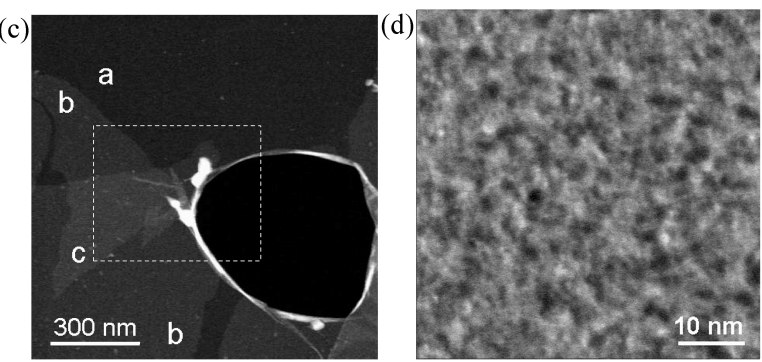

Figure 1. (a) AFM image of the GO films. (b) Histogram of the AFM-depth intensities obtained from dotted area of the image (a). (c) STEM-ADF image of the GO films where mono-, bi- and trilayers are labeled as a, b, and c, respectively. (d) High-magnification ADF image of monolayer GO film.
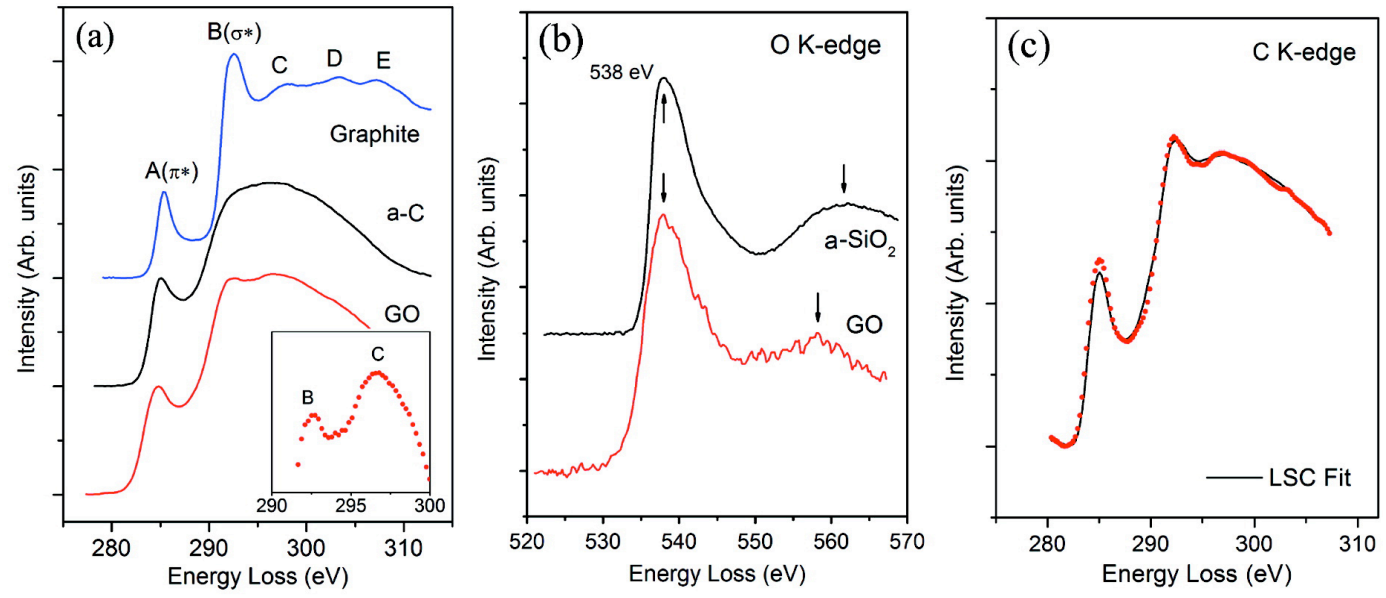

Figure 2. Core-loss EELS spectra of (a) C K-edge and (b) O K-edge from the GO film. Both spectra were recorded from the film labeled a in Fig. 1(c). The spectrum of $\mathrm{C}$ K-edge is presented in comparison with $\mathrm{C}$ Kedges measured in graphite and a-C recorded using the same STEM and similar electron-optical conditions. The peaks of the fine structure are labeled A-E. The inset shows section of the spectrum from GO for better visibility of the positions of the peaks B (or $\sigma^{*}$ ) and C. (c) The spectrum of the C K-edge in GO film and its best fit after removal of STEM probe function. 